\title{
Hamiltonian dynamics of extended objects
}

\author{
R Capovilla†, J Guven†§and E Rojas $\llbracket$ \\ † Departamento de Física, Centro de Investigación y de Estudios Avanzados del \\ IPN, Apdo Postal 14-740, 07000 México, D. F., MEXICO \\ $\ddagger$ School of Theoretical Physics, Dublin Institute for Advanced Studies, 10 \\ Burlington Road, Dublin 4, IRELAND \\ $\S$ Instituto de Ciencias Nucleares, Universidad Nacional Autónoma de México, \\ Apdo. Postal 70-543, 04510 México, DF, MEXICO \\ ๆ Facultad de Física e Inteligencia Artificial, Universidad Veracruzana, 91000 \\ Xalapa, Veracruz, MEXICO
}

\begin{abstract}
We consider a relativistic extended object described by a reparametrization invariant local action that depends on the extrinsic curvature of the worldvolume swept out by the object as it evolves. We provide a Hamiltonian formulation of the dynamics of such higher derivative models which is motivated by the ADM formulation of general relativity. The canonical momenta are identified by looking at boundary behavior under small deformations of the action; the relationship between the momentum conjugate to the embedding functions and the conserved momentum density is established. The canonical Hamiltonian is constructed explicitly; the constraints on the phase space, both primary and secondary, are identified and the role they play in the theory described. The multipliers implementing the primary constraints are identified in terms of the ADM lapse and shift variables and Hamilton's equations shown to be consistent with the Euler-Lagrange equations.
\end{abstract}

PACS numbers: 87.16.Dg, 46.70.Hg

\section{Introduction}

Relativistic extended objects, such as strings and membranes, can be viewed either as fundamental building blocks of field theories, or as providing, in their own right, field theories with an intrinsically geometric content. The geometry of interest is the trajectory, or worldvolume, swept out by the relativistic object in its evolution in a fixed background spacetime. The dynamics of this object is described by a local action constructed using the geometric scalars that characterize the worldvolume geometry. The dual requirements of worldvolume reparametrization invariance and invariance under the ambient spacetime symmetries place severe limits on the possible scalars that can be constructed. At lowest order, there is the Dirac-Nambu-Goto [DNG] action, proportional to the area of the worldvolume [1, 2, 3. This action is of first order in derivatives of the field variables - the embedding functions for the worldvolume. There are many contexts, however, where it appears that the DNG action is not adequate, and it is natural to consider models that depend on higher derivatives of these variables. Examples include the addition of a rigidity term in an effective action for stringy QCD [4, 5], the systematic approximations that arise in expansions in the thickness of topological defects $6,7,8,9$, or actions that appear in braneworld 
scenarios [10]. The dependence on derivatives of the embedding functions higher than first can appear only through the extrinsic and intrinsic curvatures of the worldvolume and their covariant derivatives. It is natural to cast the model in terms of these curvatures. Models of this kind also show up in the more formal context of non-linear physics in the description of integrable surfaces [11. Their Euclidean counterparts are also of interest as fluctuating surfaces [12, 13], and in soft condensed matter physics, e.g. in the theoretical description of lipid vesicles [14, 15.

In this paper, we examine the Hamiltonian formulation of the dynamics of relativistic extended objects described by an action that depends on the extrinsic curvature of its worldvolume. A dependence of the action on the worldvolume intrinsic curvature can always be reduced to one depending on the appropriate combinations of the extrinsic curvature using the Gauss-Codazzi equations. Of course, this topic has been explored before for particular models (see e.g. [16, 17]). However, a thorough canonical analysis is still missing. Previous approaches have typically exploited auxiliary variables, artificially reducing the higher derivative theory to an action that depends at most on first derivatives of an augmented set of field variables. This strategy has been valuable in the perturbative evaluation of functional integrals for geometric models [18. At the Hamiltonian level, however, it has the drawback of introducing artificial secondary constraints that obscure the canonical structure. It is all too easy to lose sight of the geometrical object one started out with. Another approach has been focused on the general structure of diffeomorphism invariant actions [19, 20, 21, 22, but it does not address the peculiar issues of higher derivative models. Covariant symplectic formulations of geometric models for extended objects have been examined in 23, 24].

Our approach is to grapple directly with the higher derivative nature of geometric models that depend on the extrinsic curvature. In the Hamiltonian formulation, the focus is shifted from the worldvolume geometry to the time-dependent geometry of the extended object. This approach will complement the Lagrangian approach taken in 25] (an alternative treatment of the dynamics of extended objects that emphasizes covariance with respect to the ambient spacetime has been advocated by Carter [26]).

It is first necessary to develop an appropriate geometric language to accommodate the arbitrariness in the foliation of the worldvolume by the object as it evolves. To do this, we are guided by the ADM formulation of canonical general relativity [27, where spacetime is constructed by the evolution of three-dimensional spatial slices. The ambiguity inherent in the description of the evolution is captured by the normal and tangential projections of the velocity vector: the lapse and shift variables.

We start by considering in Sect. 2 the Hamiltonian formulation of a DNG model. In order to minimize formalism, initially we restrict our attention to a relativistic object of dimension $d$ propagating in a flat Minkowski spacetime of dimension $d+2$; the worldvolume of dimension $d+1$ is a hypersurface with a single normal. The generalization to higher co-dimension is straightforward. The case of a relativistic string with $d=1$ is quite special; since it is one-dimensional its intrinsic geometry is trivial. The DNG action is a useful toy model in that it provides a benchmark for various features of the formalism which we will develop; in this case the relationship between the geometry of the worldvolume and that of the object is particularly simple. The phase space is given by the embedding functions of the object at 'fixed time' and its conjugate momentum, proportional to the timelike unit normal from the object onto the worldvolume; an immediate generalization of the momentum for a massive relativistic particle. Because of reparametrization invariance, the Hamiltonian 
vanishes; it is a linear combination of constraints. The constraints are first class and they are the generators of worldvolume reparametrizations. Next, in Sect. 3, we illustrate the main features of the general Hamiltonian formulation for an extended object with a dependence on second derivatives of the field variables. At face value this is a straightforward generalization of the classical Ostrogradski Hamiltonian treatment of higher derivative theories to extended objects [28]: in short partial derivatives get promoted to functional derivatives; the phase space is extended to include the velocity of the embedding functions for the extended object and its conjugate momentum; the Hamiltonian is given by a Legendre transformation with respect to both velocities and accelerations. There are subtleties, however, even at this level. We will show how the introduction of the ADM variables facilitates the identification of the momenta as well as the implementation of the Lagrange transformation in the construction of the Hamiltonian. Of course, the principal subtlety is the identitication of the constraints on the canonical variables associated with the underlying reparametrization invariance and understanding the role they play in the theory. For clarity, we have opted not to treat these issues in full generality, addressing them only for a class of "nondegenerate' models in which the momentum conjugate to the velocity may be inverted for the acceleration, and in particular, for the model quadratic in the mean extrinsic curvature. A model in which the dependence on the acceleration of the action is only linear, such as a model linear in the mean extrinsic curvature, behaves very differently; we will consider it elsewhere. In order to identify the extended phase space for these models, in Sect. 4 we consider the first variation of the action in a geometrical way. By examining the boundary behaviour of the varied action, we identify both the momenta conjugate to the embedding functions and those conjugate to their velocities. The latter is always normal to the worldvolume. The former is a sum of a bulk (curvature dependent) term and a total spatial divergence. In Sect. 5, we compare the structure of the canonical momenta with the conserved linear and angular momentum associated with Poincaré symmetry 29]. In Sect. 6 we focus on the derivation of the canonical Hamiltonian for a model quadratic in the mean extrinsic curvature, and we identify the primary constraints. Together with the canonical Hamiltonian these constraints generate the dynamics. There are secondary constraints; one of these is the vanishing of the canonical Hamiltonian. The secondary constraints are the generators of worldvolume diffeomorphisms. There are no tertiary constraints, and the constraints are in involution; they are first class. In Sect. 7 we check explicitly that Hamilton's equations for the model quadratic in the mean extrinsic curvature are completely equivalent to the vanishing of its Euler-Lagrange derivative. We obtain explicit expressions for the Lagrange multipliers that enforce the constraints. Unlike the DNG model, they are related in a non-trivial way to the ADM lapse and shift variables, a feature which complicates the comparison between the Lagrangian and Hamiltonian formulations of the theory. In Sect. 8 we lift the restriction to co-dimension one for the worldvolume, and we show that the Hamiltonian formulation is essentially unchanged. We conclude in Sect. 9 with some remarks. We have collected in Appendix A some useful formulas describing the geometries of the worldvolume and of the extended object. In Appendix B, we give the full Poisson algebra of the constraints for the DNG model and for the model quadratic in the mean extrinsic curvature.

In this paper, we will assume that the extended object has no spatial boundary. 


\section{Dirac-Nambu-Goto}

We consider a relativistic extended object $\Sigma$, of dimension $d$, in a fixed background Minkowski spacetime of dimension $d+2$. As $\Sigma$ evolves, it describes its trajectory or worldvolume $m$, of dimension $d+1$. We will be concerned with both the geometries of $\Sigma$ and $m$; some notational clutter is unavoidable. The worldvolume $m$ is given by the timelike embedding

$$
x^{\mu}=X^{\mu}\left(\xi^{a}\right)=X^{\mu}\left(t, u^{A}\right),
$$

where $x^{\mu}$ are local coordinates for $M^{d+2}(\mu, \nu, \ldots=0,1, \cdots, d+1), \xi^{a}$ local coordinates for the worldvolume $m(a, b, \ldots=0,1,2, \cdots, d)$, and $X^{\mu}$ are the embedding functions for $m$. In the second equality, we split the local coordinates for $m$ in an arbitrary evolution parameter $t$ and the coordinates $u^{A}$ for $\Sigma$ at fixed values of $t(A, B, \ldots=$ $1,2, \cdots, d)$. In spirit and in many technical details, this split is similar to the ADM approach to canonical general relativity (see e.g. 27]). Note that the object $\Sigma$ can be presented in two ways: as the spacelike embedding in spacetime $x^{\mu}=X^{\mu}\left(t=0, u^{A}\right)$, or as the spacelike embedding in the worldvolume $m, \xi^{a}=X^{a}\left(u^{A}\right)$, related by composition. Recall that we assume that the extended object $\Sigma$ has no spatial boundary. (We have collected some useful formulae describing the geometries of $m$ and $\Sigma$ in Appendix A.)

In this section, we focus on the Hamiltonian formulation of an object whose dynamics is determined by the DNG action, proportional to the area of the worldvolume swept out by the object in its evolution (see e.g. 31, 32, 33]). The basic field variables are the embedding functions for the worldvolume, and this action depends only on first derivatives of the field variables. The DNG action is the generalization to extended objects of the action for a relativistic particle in its geometrical square root form. The action is

$$
S[X]=-\mu \int_{m} d^{d+1} \xi \sqrt{-g}=-\mu \int_{m} \sqrt{-g} .
$$

Henceforth, we will absorb the differentials $d^{d+1} \xi$ in the integral sign. The constant $\mu$ is the tension. $g$ denotes the determinant of the induced metric on $m$,

$$
g_{a b}=\eta_{\mu \nu} X_{a}^{\mu} X_{b}^{\nu}=X_{a} \cdot X_{b},
$$

where $X_{a}=\partial X / \partial \xi^{a}$ are the $d+1$ tangent vectors to $m$, and $\eta_{\mu \nu}$ is the Minkowski metric with one minus sign. We will denote by $\nabla_{a}$ the worldvolume covariant derivative compatible with $g_{a b}$. Latin indices are lowered and raised with $g_{a b}$ and its inverse $g^{a b}$, respectively.

The first step towards a Hamiltonian formulation is to consider the first variation of the action under an infinitesimal deformation of the embedding functions $X \rightarrow$ $X+\delta X$. We readily obtain

$$
\begin{aligned}
\delta S[X] & =-\mu \int_{m} \sqrt{-g} g^{a b} X_{a} \cdot \partial_{b} \delta X \\
& =\mu \int_{m} \partial_{b}\left[\sqrt{-g} g^{a b} X_{a}\right] \cdot \delta X-\mu \int_{m} \partial_{b}\left[\sqrt{-g} g^{a b} X_{a} \cdot \delta X\right] .
\end{aligned}
$$

We have used the fact that $\delta X_{a}=\partial_{a} \delta X$, and we have integrated by parts to obtain the second line. We note that, using Stokes' theorem, for an arbitrary worldvolume vector $V^{a}$, we have

$$
\int_{m} \partial_{a}\left(\sqrt{-g} V^{a}\right)=-\int_{\Sigma} d^{d} u \sqrt{h} \eta_{a} V^{a}=-\int_{\Sigma} \sqrt{h} \eta_{a} V^{a} .
$$


Here $\eta^{a}$ is the timelike unit normal to $\Sigma$ onto $m, g_{a b} \eta^{a} \eta^{b}=-1$, and the minus sign on the right hand side of (5) comes about because of the direction of the normal $\eta ; h$ denotes the determinant of the metric induced on $\Sigma$,

$$
h_{A B}=X_{A} \cdot X_{B}=g_{a b} X_{A}^{a} X_{B}^{b}
$$

with either $X_{A}=\partial X / \partial u^{A}$ or $X_{A}^{a}=\partial X^{a} / \partial u^{A}$ the $d$ tangent vectors to $\Sigma$. Capital latin indices are lowered and raised with $h_{A B}$ and its inverse $h^{A B}$, respectively. We will denote with $\mathcal{D}_{A}=X_{A}^{a} \nabla_{a}$ the spatial covariant derivative on $\Sigma$, compatible with $h_{A B}$. Note that $g_{a b} \eta^{a} X_{A}^{b}=0$. The worldvolume vectors $\left\{\eta^{a}, X_{A}^{a}\right\}$ form a basis for $m$ adapted to $\Sigma$. They satisfy the completeness relation

$$
g^{a b}=-\eta^{a} \eta^{b}+h^{A B} X_{A}^{a} X_{B}^{b} .
$$

One convenient way to obtain the canonical momentum $p$ conjugate to the embedding functions $X$ is to recall that the first variation of the action can be written, for a first order theory, as

$$
\delta S[X]=\int_{m} \sqrt{-g} E \cdot \delta X+\int_{\Sigma} p \cdot \delta X,
$$

where $E$ denotes the Euler-Lagrange derivative of $S[X]$. From (44) and (5), we can cast the first variation of the action in the form (8) with

$$
\delta S[X]=\mu \int_{m} \partial_{b}\left[\sqrt{-g} g^{a b} X_{a}\right] \cdot \delta X+\mu \int_{\Sigma} \sqrt{h} \eta_{b} g^{a b} X_{a} \cdot \delta X,
$$

so that the canonical momenta can be read off from the boundary term as

$$
p=\mu \sqrt{h} \eta^{a} X_{a}=\mu \sqrt{h} \eta .
$$

It is proportional to the timelike unit normal to $\Sigma$ into the worldsheet. We write $\eta=\eta^{a} X_{a} ; p$ is a spatial density of weight one. Note that in the limit of a pointlike object we have that $h=1, \mu$ is the mass, and $\eta$ the relativistic velocity.

We have thus identified the phase space for the DNG model: the embedding functions for the relativistic object $\Sigma$ are the canonical coordinates, and their conjugate canonical momenta are proportional to the densitized timelike unit normal to $\Sigma$ into the worldvolume.

From (9), we can also identify the Euler-Lagrange derivative of the DNG action as

$$
E=-\mu \frac{1}{\sqrt{-g}} \partial_{b}\left[\sqrt{-g} g^{a b} X_{a}\right]=-\mu \nabla^{2} X=-\mu K n,
$$

where $\nabla^{2}=g^{a b} \nabla_{a} \nabla_{b}$ denotes the d'Alembert operator, and $K$ is the mean extrinsic curvature, defined by $K=g^{a b} K_{a b}$ with

$$
K_{a b}=-n \cdot \partial_{a} X_{b}
$$

the extrinsic curvature of $m$, and $n$ the spacelike unit normal to $m$, defined implicitly by $n \cdot X_{a}=0, n \cdot n=1$. It follows that the equations of motion for a DNG object can be expressed succintly as the vanishing of the mean extrinsic curvature

$$
-\mu K=0 \text {. }
$$

We obtain the canonical Hamiltonian via a Legendre transformation with respect to the velocity of the embedding functions for $\Sigma$,

$$
H_{c}[X, p]=\int_{\Sigma} p \cdot \dot{X}-L[X, \dot{X}] .
$$


The velocity $\dot{X}=\partial_{t} X=\partial_{0} X$ is a vector tangent to the worldvolume $m$ (note that we were able to identify $p$ without any explicit reference to $\dot{X}$ ). It can be expanded in components with respect to the basis of tangent vectors to the worldvolume, $\left\{\eta, X_{A}\right\}$ adapted to $\Sigma$ as

$$
\dot{X}=N \eta+N^{A} X_{A} .
$$

Using a language borrowed from canonical general relativity, the projections of $\dot{X}$ onto this basis are the lapse function $N$ and the shift vector $N^{A}$. In this model, neither $N$ nor $N^{A}$ is a canonical variable; in the higher derivative theory we will see that they both are.

With respect to the coordinate basis $\left\{\dot{X}, X_{A}\right\}$ adapted to the evolution, the worldvolume metric (3) takes its ADM form

$$
g_{a b}=\left(\begin{array}{cc}
-N^{2}+N^{A} N^{B} h_{A B} & h_{A B} N^{B} \\
h_{A B} N^{B} & h_{A B}
\end{array}\right),
$$

and its determinant is given by

$$
g=-N^{2} h,
$$

in terms of the lapse function $N$ and the determinant $h$ of the spatial metric $h_{A B}$. We will also need the ADM form of the inverse metric,

$$
g^{a b}=\frac{1}{N^{2}}\left(\begin{array}{cc}
-1 & N^{A} \\
N^{A} & N^{2} h^{A B}-N^{A} N^{B}
\end{array}\right) .
$$

The DNG Lagrangian functional is

$$
S=\int d t L[X, \dot{X}]
$$

where

$$
L[X, \dot{X}]=-\mu \int_{\Sigma} N \sqrt{h},
$$

and we have used (17). Note that the canonical momenta can also be obtained directly by the functional derivative $p=\delta L / \delta \dot{X}=\mu \sqrt{h} \eta$, since $N=-\eta \cdot \dot{X}$, in agreement with (10). However, this direct derivation becomes difficult to carry out for higher derivative actions.

Using (10), the canonical Hamiltonian is given by

$$
H_{c}[X, p]=\int_{\Sigma}[\mu \sqrt{h} \eta \cdot \dot{X}+\mu N \sqrt{h}]=0 .
$$

It vanishes; this was to be expected because of the reparametrization invariance of the DNG action. How this comes about is rather more subtle in the higher derivative theory. According to the standard Dirac-Bergmann theory of constrained systems, the Hamiltonian is a linear combination of constraints, which are easily identified from the definition of the momenta (10) as

$$
\begin{aligned}
& C_{A}=p \cdot X_{A}=0, \\
& C=p^{2}+\mu^{2} h=0 .
\end{aligned}
$$

Note that $C_{A}$ is a spatial scalar density of weight one, whereas $C$ is a spatial scalar density of weight two. There are $d+1$ constraints. It is easy to check that they are in involution, so they are first class constraints (see Appendix B). They are the generators of worldvolume reparametrizations; $C_{A}$ generates diffeomorphisms 
tangential to $\Sigma$, whereas $C$ generates diffeomorphisms out of $\Sigma$ onto the worldvolume $m$. For a relativistic string, $h$ provides a quadratic potential in the constraint (23); for a relativistic membrane $h$ provides a quartic potential. The number of physical degrees of freedom is given by $1 / 2$ [ (total number of canonical variables) $-2 \times$ (number of first class constraints)]. We obtain that there is a single physical degree of freedom, which corresponds to motions along the single normal to the worldvolume. For higher co-dimension there will be a physical degree of freedom along each normal.

It follows that the Hamiltonian that generates the dynamics is

$$
H[X, p]=\int_{\Sigma}\left(\lambda C+\lambda^{A} C_{A}\right)
$$

where $\lambda, \lambda^{A}$ are arbitrary Lagrange multipliers that enforce the constraints. Note that $\lambda$ must be a spatial density of weight minus one for the integral over $\Sigma$ to be well-defined.

The Poisson brackets are, in terms of two arbitrary phase space functions $f, g$,

$$
\{f, g\}=\int_{\Sigma}\left(\frac{\delta f}{\delta X} \cdot \frac{\delta g}{\delta p}-\frac{\delta g}{\delta X} \cdot \frac{\delta f}{\delta p}\right) .
$$

Let us check explicitly that the Hamilton equations that follow from (24) reproduce the equations of motion (13). This exercise is useful to illustrate various features of the Hamilton equations for an extended object. The first Hamilton equation is

$$
\dot{X}=\frac{\delta H}{\delta p}=2 \lambda p+\lambda^{A} X_{A},
$$

and, as in its non-relativistic counterpart, it serves to express the momenta in terms of the velocities. Using the constraints (22), (23), the Lagrange multipliers are identified as

$$
\lambda=\frac{N}{2 \mu \sqrt{h}}, \quad \lambda^{A}=N^{A} .
$$

As we will see, this simple identification does not hold in higher order theories. With this identification, the Hamilton equation (26) reproduces the form of the canonical momenta (10). The second Hamilton equation is

$$
\begin{aligned}
\dot{p} & =-\frac{\delta H}{\delta X}=-\int_{\Sigma}\left(\lambda \mu^{2} h h^{A B} \frac{\delta h_{A B}}{\delta X}+\lambda^{A} p \cdot \frac{\delta X_{A}}{\delta X}\right), \\
& =\partial_{A}\left(2 \mu^{2} \lambda h h^{A B} X_{B}+N^{A} p\right)
\end{aligned}
$$

where we have integrated by parts, disregarded a total spatial divergence, and used

$$
\begin{aligned}
& \delta h_{A B}=2 X_{(A} \cdot \partial_{B)} \delta X, \\
& \delta h \quad=2 h h^{A B} X_{A} \cdot \partial_{B} \delta X .
\end{aligned}
$$

Use of the form (27) for the Lagrange multipliers gives the equations of motion in canonical form as

$$
\dot{p}=\partial_{A}\left(\mu N \sqrt{h} h^{A B} X_{B}+N^{A} p\right)
$$

The time derivative of the canonical momenta is given by a spatial divergence. The total momentum is conserved. This feature is common to any Poincaré invariant action. The reason is simple: because of Poincaré invariance the dependence on the embedding functions $X$ of the Hamiltonian can appear only through its spatial 
derivatives. Note that the right hand side of (31) is the divergence of a spatial density of weight one; as such, it is independent of the spatial affine connection.

This canonical expression for the equations of motion should be compared with the worldvolume covariant equations of motion (13). To see that they coincide, we decompose the DNG Euler-Lagrange derivative appearing in (11) with respect to the basis $\left\{\dot{X}, X_{A}\right\}$, adapted to the evolution of $\Sigma$. We have, using (17), (18) for $\sqrt{-g}$ and $g^{a b}$, or, alternatively,

$$
\begin{aligned}
& g^{0 b}=-\frac{1}{N} \eta^{b}, \\
& g^{A b}=h^{A B} X_{B}^{b}+\frac{N^{A}}{N} \eta^{b},
\end{aligned}
$$

that

$$
\begin{aligned}
\sqrt{-g} E & =-\mu \partial_{0}\left(N \sqrt{h} g^{0 b} X_{b}\right)-\mu \partial_{A}\left(N \sqrt{h} g^{A b} X_{b}\right) \\
& =\mu \partial_{0}(\sqrt{h} \eta)-\mu \partial_{A}\left(\sqrt{h} N^{A} \eta+N \sqrt{h} h^{A B} X_{B}\right) .
\end{aligned}
$$

Now we need only to recognize that $\mu \sqrt{h} \eta=p$, to obtain

$$
\sqrt{-g} E=\dot{p}-\partial_{A}\left(\mu N \sqrt{h} h^{A B} X_{B}+N^{A} p\right),
$$

whose vanishing coincides with the canonical form of the equations of motion (31).

\section{Higher derivatives models}

We turn now to geometric models for the dynamics of relativistic extended objects that depend, in addition to the velocity as in the DNG model, also on the acceleration of the embedding functions, $\ddot{X}$. We consider a local action of the form

$$
S[X]=\int d t L[X, \dot{X}, \ddot{X}]
$$

where $L[X, \dot{X}, \ddot{X}]$ is the Lagrangian functional. We are dealing with a higher derivatives theory. The Hamiltonian formulation of such theories [28] involves the extension of the phase space to $\{p, X ; P, \dot{X}\}$, where $P$ and $p$ denote the momenta conjugate to $\dot{X}$ and $X$, respectively. They can be obtained directly from the functional derivatives of the Lagrangian functional

$$
\begin{aligned}
& P=\frac{\delta L}{\delta \ddot{X}}, \\
& p=\frac{\delta L}{\delta \dot{X}}-\partial_{0}\left(\frac{\delta L}{\delta \ddot{X}}\right) .
\end{aligned}
$$

Alternatively, the momenta can be read off from the boundary term in the first order variation of the action, that, for a theory that depends at most on second derivatives of the field variables, can always be written in the form

$$
\delta S[X]=\int_{m} \sqrt{-g} E \cdot \delta X+\int_{\Sigma}(P \cdot \delta \dot{X}+p \cdot \delta X),
$$

where $E$ denotes the Euler-Lagrange derivative. This is the higher derivative generalization of (8).

The canonical Hamiltonian is given by the Legendre transformation with respect to both $\ddot{X}$ and $\dot{X}$ as

$$
H_{c}[X, p ; \dot{X}, P]=\int_{\Sigma}(P \cdot \ddot{X}+p \cdot \dot{X})-L[X, \dot{X}, \ddot{X}] .
$$


In this expression, it is understood that the acceleration $\ddot{X}$ is expressed in terms of the phase space variables $P, \dot{X}, X$, just as in a first order theory one expresses $\dot{X}$ in terms of $p$ and $X$. It should be emphasized that $p$ is left alone. The resulting expression for the canonical Hamiltonian is

$$
H_{c}[X, p ; \dot{X}, P]=\int_{\Sigma}(p \cdot \dot{X}+V[X, \dot{X}, P])
$$

where the form of the 'potential' $V[X, \dot{X}, P]$ will depend on the specific model under consideration.

The presence of symmetries manifests itself in the canonical formalism by the appearance of constraints. If there are primary constraints, they are identified by the null eigenvectors of the Hessian

$$
\mathcal{H}_{\mu \nu}=\frac{\delta^{2} L[X, \dot{X}, \ddot{X}]}{\delta \ddot{X}^{\mu} \delta \ddot{X}^{\nu}}
$$

and, if present, must be added to the canonical Hamiltonian (40). This produces an extended Hamiltonian which generates the dynamics via the Hamilton equations. Rather than addressing this issue in full generality here, we prefer to address it within a specific model below in Sect. 6 .

The Poisson brackets (25) generalize to

$$
\{f, g\}=\int_{\Sigma}\left[\frac{\delta f}{\delta X} \cdot \frac{\delta g}{\delta p}+\frac{\delta f}{\delta \dot{X}} \cdot \frac{\delta g}{\delta P}-(f \leftrightarrow g)\right] .
$$

Let us consider briefly the structure of the Hamilton equations. The first one is vacuous, since the only dependence of the Hamiltonian on $p$ is through the $p \cdot \dot{X}$ term, $\dot{X}=\{X, H\}=\delta H / \delta p=\dot{X}$. The second provides the definition of the momenta $P$ conjugate to $\dot{X}, \ddot{X}=\delta H / \delta P$, and it reproduces (37). Moreover, it identifies the explicit form of the Lagrange multipliers that enforce the primary constraints, if present. The third Hamilton equation provides the definition of the momenta $p$ conjugate to $X, \dot{P}=-\delta H / \delta \dot{X}$, and it reproduces (38). Finally, the fourth equation gives the equations of motion in Hamiltonian form, once the expressions for $p, P$ are used: $\dot{p}=-\delta H / \delta X$.

For a relativistic extended object, the requirement of reparametrization invariance implies that the dependence on second derivatives of the embedding functions can appear only through the extrinsic curvature of the worldvolume $m$. Therefore, the explicitly covariant form of the action (36) can be written as

$$
S[X]=\int_{m} \sqrt{-g} L\left(g^{a b}, K_{a b}\right),
$$

where $L\left(g^{a b}, K_{a b}\right)$ is a scalar constructed from the inverse induced metric and the extrinsic curvature tensor $K_{a b}=-n \cdot \partial_{a} X_{b}$. A possible dependence on the intrinsic curvature of the worldvolume $\mathcal{R}_{a b c d}$ can always be expressed in terms of the extrinsic curvature via the Gauss-Codazzi equations for the worldvolume $m$,

$$
\mathcal{R}_{a b c d}=K_{a c} K_{b d}-K_{a d} K_{b c} .
$$

An important example of an action of the form (44) is provided by the rigidity model, quadratic in the mean extrinsic curvature,

$$
S[X]=\frac{1}{2} \int_{m} \sqrt{-g} K^{2} .
$$


For a relativistic string, the addition of this term dependent on the extrinsic geometry to the DNG action has been suggested by Polyakov [4, and independently by Kleinert [5], in an effective description of QCD. Aspects of its Hamiltonian formulation have been considered by Nesterenko and Suan Han [16.

This quadratic model is representative of the generic case at least as long as the rank of the Hessian (42) does not change. What will change is the specific form of the potential $V[X, \dot{X}, P]$ in the canonical Hamiltonian (41).

One important exception is the case when the dependence on the acceleration in (44) is only linear. An example is the Tolman model, valid only for a hypersurface, linear in the mean extrinsic curvature [30]

$$
S[X]=\int_{m} \sqrt{-g} K .
$$

Another is the Einstein-Hilbert action for a relativistic extended object 34, 35, 36.

$$
S[X]=\int_{m} \sqrt{-g} \mathcal{R}
$$

which, for a relativistic string, by the Gauss-Bonnet theorem, is a topological invariant. The canonical analysis of these cases is qualitatively different. We will consider them elsewhere [37. The problem is that it is not possible to carry out the Legendre transformation, i.e. invert (37) to express $\ddot{X}$ in terms of the extended phase space variables.

\section{First variation and momenta}

In this section, we use the first variation of the action to identify both the equations of motion and the canonical momenta conjugate to $\{X, \dot{X}\}$ for the extrinsic curvature dependent action (44). For the latter purpose, one approach would be to carry out directly the functional derivatives of the Lagrangian functional as in the definitions (37), (38). However, this turns out to be quite difficult to do. The problem is that the dependence on the velocity $\dot{X}$ of the Lagrangian functional $L[X, \dot{X}, \ddot{X}]$ is difficult to pinpoint. A more convenient strategy is to consider the covariant first variation of the action (44) in the form (39), and read out the momenta from the boundary contribution, just as we did for the DNG model in Sect. 2.

Consider an infinitesimal deformation $X \rightarrow X+\delta X$ of the embedding functions for the worldlvolume $m$. Under this deformation, to first order, the basic geometric quantities that characterize the worldvolume $m$ change according to

$$
\begin{aligned}
& \delta g_{a b}=2 X_{(a} \cdot \nabla_{b)} \delta X, \\
& \delta g^{a b}=-2 X^{(a} \cdot \nabla^{b)} \delta X, \\
& \delta \sqrt{-g}=\sqrt{-g} g^{a b} X_{a} \cdot \nabla_{b} \delta X, \\
& \delta K_{a b}=-n \cdot \nabla_{a} \nabla_{b} \delta X .
\end{aligned}
$$

Using these expressions in the first variation of the action (44), we have

$$
\begin{aligned}
\delta S[X] & =\int_{m}\left[(\delta \sqrt{-g}) L+\sqrt{-g}\left(H_{a b} \delta g^{a b}+L^{a b} \delta K_{a b}\right)\right. \\
& =\int_{m} \sqrt{-g}\left[L g^{a b} X_{a} \cdot \nabla_{b} \delta X-2 H_{a b} X^{a} \cdot \nabla^{b} \delta X-L^{a b} n \cdot \nabla_{a} \nabla_{b} \delta X\right],
\end{aligned}
$$


where we have defined

$$
H_{a b}=\frac{\partial L}{\partial g^{a b}}, \quad L^{a b}=\frac{\partial L}{\partial K_{a b}} .
$$

Note that they are related by the identity [29]

$$
2 H^{a b}=L^{a c} K_{c}{ }^{b}+L^{b c} K_{c}{ }^{a} .
$$

In order to cast the first variation of the action (53) in the form (39), we integrate (53) by parts, and we obtain

$$
\delta S=\int_{m} \sqrt{-g}\left(E \cdot \delta X+\nabla_{b} Q^{b}\right),
$$

where we have defined the quantity appearing in the total divergence

$$
Q^{b}=\left[g^{a b} L X_{a}-2 H^{a b} X_{a}+\nabla_{a}\left(L^{a b} n\right)\right] \cdot \delta X-L^{a b} n \cdot \nabla_{a} \delta X .
$$

Note that $Q^{a}$ depends on the infinitesimal deformation $\delta X$ and its first derivative. If we had allowed for a dependence of the action on derivatives of the extrinsic curvature, then $Q^{a}$ would depend also on second derivatives of the deformation.

In (56), $E$ is Euler-Lagrange derivative of the action (44)

$$
\begin{aligned}
E & =-\nabla_{b}\left(g^{a b} L X_{a}\right)+2 \nabla_{b}\left(H_{a b} X^{a}\right)-\nabla_{b} \nabla_{a}\left(L^{a b} n\right) \\
& =\left(-\nabla_{b} \nabla_{a} L^{a b}+K L-L^{a b} K_{a}{ }^{c} K_{b c}\right) n,
\end{aligned}
$$

where we have used the Gauss-Weingarten equations for the worldvolume

$$
\begin{aligned}
& \nabla_{a} X_{b}=-K_{a b} n, \\
& \nabla_{a} n=K_{a b} g^{b c} X_{c},
\end{aligned}
$$

together with the identity (55) to obtain the second line. The Euler-Lagrange derivative is purely normal; this is a consequence of reparametrization invariance.

For the quadratic model (46), with $H_{a b}=K K_{a b}$, and $L^{a b}=K g^{a b}$, its EulerLagrange derivative takes the form

$$
\sqrt{-g} E=\partial_{b}\left\{\sqrt{-g}\left[-\frac{1}{2} g^{a b} K^{2} X_{a}+2 K K^{a b} X_{a}-g^{a b} \nabla_{a}(K n)\right]\right\},
$$

where we have absorbed the factor of $\sqrt{-g}$ in order to facilitate the comparison with Hamilton's equations later on.

In the canonical analysis, to arrive at the momenta, we focus on the boundary term in (56), involving $Q^{a}$. We have, using Stokes' theorem (5), that

$$
\begin{aligned}
\int_{m} \sqrt{-g} \nabla_{b} Q^{b} & =-\int_{\Sigma} \sqrt{h} \eta_{b} Q^{b} \\
& =-\int_{\Sigma} \sqrt{h}\left[L \eta-2 \eta_{b} H^{a b} X_{a}+\eta_{b}\left(\nabla_{a} L^{a b} n\right)\right] \cdot \delta X \\
& +\int_{\Sigma} \sqrt{h} \eta_{b} L^{a b} n \cdot \nabla_{a} \delta X .
\end{aligned}
$$

Comparison with (39) shows that the second line will give a contribution to the canonical momenta $p$ conjugate to $X$. However, the dependence on $\delta \dot{X}$ is only implicit in the last term of (63). In order to isolate it, we consider an arbitrary worldvolume vector $V^{a}$, and we exploit the completeness relation on the worldvolume (7) to get

$$
\begin{aligned}
V^{a} n \cdot \nabla_{a} \delta X & =V_{a} g^{a b} n \cdot \nabla_{b} \delta X \\
& =-V_{a} \eta^{a} \eta^{b} n \cdot \nabla_{b} \delta X+V_{b} X^{b A} n \cdot \partial_{A} \delta X \\
& =-\frac{1}{N} V_{b} \eta^{b} n \cdot \delta \dot{X}+\left(\frac{1}{N} V_{b} \eta^{b} N^{A}+V_{b} X^{b A}\right) n \cdot \partial_{A} \delta X .
\end{aligned}
$$


We now set $V^{a}=\sqrt{h} \eta_{b} L^{a b}$, and use this expression in the last term of (63) to obtain

$$
\begin{aligned}
\int_{\Sigma} & \sqrt{h} \eta_{b} L^{a b} n \cdot \nabla_{a} \delta X \\
& =\int_{\Sigma}\left[-\frac{\sqrt{h}}{N} \eta_{b} L^{a b} \eta_{a} n \cdot \delta \dot{X}+\frac{\sqrt{h}}{N}\left(\eta_{a} L^{a b} \eta_{b} N^{A}+N \eta^{a} L_{a b} X^{b A}\right) n \cdot \partial_{A} \delta X\right] \\
& =\int_{\Sigma}\left\{-\frac{\sqrt{h}}{N} \eta_{b} L^{a b} \eta_{a} n \cdot \delta \dot{X}-\partial_{A}\left[\frac{\sqrt{h}}{N}\left(\eta_{a} L^{a b} \eta_{b} N^{A}+N \eta^{a} L_{a b} X^{b A}\right) n\right] \cdot \delta X\right\}(65)
\end{aligned}
$$

We have integrated by parts and disregarded a total spatial divergence. We see that the boundary term which involves a derivative of the variation identifies the momenta $P$ conjugate to $\dot{X}$ together with a contribution to the momenta $p$ given by a spatial divergence. The appearance of this latter term is a novelty introduced by the Hamiltonian formulation.

Using (65) in (63), comparison with (39) produces the canonical momenta $P, p$ conjugate to $\dot{X}, X$, respectively, as

$$
\begin{gathered}
P=-\frac{\sqrt{h}}{N} \eta_{b} L^{a b} \eta_{a} n, \\
p=-\sqrt{h}\left[L \eta-2 \eta_{b} H^{a b} X_{a}+\eta_{b} \nabla_{a}\left(L^{a b} n\right)\right]+\partial_{A}\left[N^{A} P-\sqrt{h} \eta^{a} L_{a b} X^{b A} n\right] .
\end{gathered}
$$

The momenta $P$ conjugate to $\dot{X}$ is always normal to the worldvolume, and it vanishes if the action does not depend on the extrinsic curvature. The momenta $p$ conjugate to $X$ is given by two parts, with the second a spatial divergence. The first part is related to the conserved linear momentum for this system. We will analyze this relationship in the next section. Note that both $P$ and $p$ are spatial densities of weight one; their spatial derivative is independent of the spatial affine connection. This fact will be used extensively below. Note also how the case of a DNG model is quite special: only the first term in (67) survives, and $p$ is purely tangential to the worldvolume.

The momenta $p$ conjugate to $X$ can be written in an alternative way using the Gauss-Weingarten equation (61) together with the identity (55), as

$p=-\sqrt{h}\left[L \eta-\eta_{b} L^{a c} K^{b}{ }_{c} X_{a}+\eta_{b}\left(\nabla_{a} L^{a b}\right) n\right]+\partial_{A}\left[N^{A} P-\sqrt{h} \eta^{a} L_{a b} X^{b A} n\right]$.

If we specialize our considerations to the quadratic model (46), then the momenta (66), (67) take the form

$$
\begin{aligned}
& P=\frac{\sqrt{h}}{N} K n \\
& p=\sqrt{h}\left[-\frac{1}{2} K^{2} \eta+2 \eta_{b} K K^{a b} X_{a}-\eta^{a} \nabla_{a}(K n)\right]+\partial_{A}\left(N^{A} P\right) .
\end{aligned}
$$

This concrete example makes it clear how the higher derivative nature of the model determine the structure of the canonical momenta $P, p$.

\section{Conservation laws}

The conservation laws associated with the Poincaré invariance for geometric models of relativistic objects were examined in [29], exploiting Noether's theorem. This made it possible to derive general expressions for both the linear and angular momenta for any action of the form (44) depending on the extrinsic curvature. In this section, 
we examine the relationships between these conserved quantities and the canonical momenta.

Return to the first variation of the action in the form (56). If we specialize the variation of the embedding functions to an infinitesimal constant translation $\delta X=\epsilon$ and we set $Q^{a}=-\epsilon_{\mu} T^{\mu a}$ (we refer to [29] for details), we obtain for the energymomentum tensor associated with the action (44),

$$
T^{\mu a}=\left(L g^{a b}-L^{b c} K^{a}{ }_{c}\right) X_{b}^{\mu}+\nabla_{b}\left(L^{a b}\right) n^{\mu} .
$$

In particular, for an action that is invariant under translations, it follows that we can write the Euler-Lagrange derivative as

$$
E^{\mu}=\nabla_{a} T^{\mu a} .
$$

Let us define the linear momentum density associated with the object $\Sigma$ by

$$
\pi^{\mu}=\sqrt{h} \eta_{a} T^{\mu a} .
$$

Then we see that it differs from the canonical momenta $p$ conjugate to $X$ (67) by a total spatial divergence,

$$
p=\pi+\partial_{A}\left[N^{A} P-\sqrt{h} \eta^{a} L_{a b} X^{b A} n\right] .
$$

In particular, it follows that both $p$ and $\pi$ give the same total linear momentum of the object $\Sigma$ since

$$
\int_{\Sigma} p=\int_{\Sigma} \pi
$$

up to a vanishing boundary term.

The boundary term is related to the angular momentum asociated with the action (44). If we specialize the variation of the embedding functions to an infinitesimal Lorentz transformation $\delta X^{\mu}=\omega^{\mu}{ }_{\nu} X^{\nu}$, with $\omega_{\mu \nu}=-\omega_{\nu \mu}$, and we set $Q^{a}=$ $-\omega_{\mu \nu} M^{\mu \nu a}$, we obtain for the angular momentum

$$
\begin{aligned}
M^{\mu \nu a} & =\frac{1}{2}\left[T^{\mu a} X^{\nu}+L^{a b} n^{\mu} X_{b}^{\nu}-(\mu \leftrightarrow \nu)\right] \\
& =\frac{1}{2}\left[T^{\mu a} X^{\nu}-L^{a b} \eta_{b} n^{\mu} \eta^{\nu}+L^{a b} X_{b}^{A} n^{\mu} X_{A}^{\nu}-(\mu \leftrightarrow \nu)\right],
\end{aligned}
$$

where in the second line we have decomposed $X_{b}$ along the basis $\left\{\eta, X_{A}\right\}$. The angular momentum is given by the sum of an orbital part, that depends on the origin, and a differential part. The latter is related to the boundary term that appears in (67).

\section{Hamiltonian and constraints}

In this section, we restrict our attention to the model quadratic in the mean extrinsic curvature (46), since the generic case given by (44) can be treated along the same lines. We derive the canonical Hamiltonian (40) and we identify the constraints on the phase space variables that follow from the reparametrization invariance of the action.

The canonical Hamiltonian is given by the Legendre transformation with respect to both $\ddot{X}$ and $\dot{X}$ (see (40)),

$$
H_{c}[X, p ; \dot{X}, P]=\int_{\Sigma}(P \cdot \ddot{X}+p \cdot \dot{X})-L[X, \dot{X}, \ddot{X}] .
$$


Using (69) for the higher momenta $P$, we have immediately that the Lagrangian functional can be expressed in terms of the phase space variables as

$$
L[X, \dot{X}, \ddot{X}]=\frac{1}{2} \int_{\Sigma} N \sqrt{h} K^{2}=\frac{1}{2} \int_{\Sigma} \frac{N^{3}}{\sqrt{h}} P^{2} .
$$

In order to carry out the Legendre transformation, we need to express the acceleration $\ddot{X}$ in terms of $\{P, \dot{X}, X\}$. Recall that $p$ is left alone. With (69) for the momenta $P$, we have

$$
P \cdot \ddot{X}=\frac{\sqrt{h}}{N} K n \cdot \ddot{X} .
$$

We need to write the normal component of the acceleration in terms of the extrinsic curvature. For this purpose, note that it is given by (minus) the time-time projection of the extrinsic curvature

$$
K_{00}=\dot{X}^{a} \dot{X}^{b} K_{a b}=-n \cdot \ddot{X} .
$$

The other projections of the extrinsic curvature are

$$
\begin{aligned}
& K_{0 A}=\dot{X}^{a} X_{A}^{b} K_{a b}=-n \cdot \partial_{A} \dot{X}, \\
& K_{A B}=X_{A}^{a} X_{B}^{b} K_{a b}=-n \cdot \mathcal{D}_{A} \mathcal{D}_{B} X .
\end{aligned}
$$

We use the definition of the velocity $\dot{X}$ (15), together with the worldvolume completeness relation (17) to obtain for the time-time projection of the extrinsic curvature (79),

$$
\begin{aligned}
K_{00} & =N^{2} \eta^{a} \eta^{b} K_{a b}+2 N N^{A} \eta^{a} X_{A}^{b} K_{a b}+N^{A} N^{B} K_{A B} \\
& =-N^{2} K+2 N N^{A} \eta^{a} X_{A}^{b} K_{a b}+\left(N^{2} h^{A B}+N^{A} N^{B}\right) K_{A B} \\
& =-N^{2} K+2 N N^{A} K_{0 A}+\left(N^{2} h^{A B}-N^{A} N^{B}\right) K_{A B},
\end{aligned}
$$

therefore from (78) we have

$$
P \cdot \ddot{X}=\frac{\sqrt{h}}{N}\left[N^{2} K^{2}-2 N^{A} K K_{0 A}-\left(N^{2} h^{A B}-N^{A} N^{B}\right) K K_{A B}\right],
$$

and using once more (69) for $P$, we find that $P \cdot \ddot{X}$ is expressed in terms of the phase space variables by

$$
P \cdot \ddot{X}=\frac{N^{3}}{\sqrt{h}} P^{2}+2 N^{A} P \cdot \partial_{A} \dot{X}+\left(N^{2} h^{A B}-N^{A} N^{B}\right) P \cdot \mathcal{D}_{A} \mathcal{D}_{B} X .
$$

Using (77) for the Lagrangian functional and this last expression in the Legendre transformation (40) gives the Hamiltonian as

$H_{c}[X, p ; \dot{X}, P]=\int_{\Sigma}\left[p \cdot \dot{X}+\frac{N^{3}}{2 \sqrt{h}} P^{2}+2 N^{A} P \cdot \partial_{A} \dot{X}+\left(N^{2} h^{A B}-N^{A} N^{B}\right) P \cdot \mathcal{D}_{A} \mathcal{D}_{B} X\right]$

The dependence of this Hamiltonian on $P, p, \dot{X}$ is explicit. On the other hand, the dependence on $X$ is not so evident. Besides the dependence on $X$ through the induced metric on $\Sigma, h_{A B}$, and the last term, where $X$ appears explicitly, there is also a dependence on $X$ of both the lapse function and the shift vector $N, N^{A}$. We will address this technical point explicitly in the next section.

Note that for a point-like object, this Hamiltonian specializes to

$$
H_{c}[X, p ; \dot{X}, P]=p \cdot \dot{X}+\frac{N^{3}}{2} P^{2} .
$$


This agrees with the Hamiltonian obtained e.g. in 38 for a relativistic particle model with an action quadratic in the geodesic curvature.

Just as in the case of a DNG extended object, reparametrization invariance of the action (46) implies the existence of constraints. According to the Dirac-Bergmann theory of higher derivative theories, we immediately identify the primary constraints that involve the highest momenta $P$ from its definition as

$$
\begin{aligned}
& C=P \cdot \dot{X}=0, \\
& C_{A}=P \cdot X_{A}=0 .
\end{aligned}
$$

There are no other primary constraints involving $P$, as one can check by computing the Hessian (42)

$$
H_{\mu \nu}=\frac{1}{N^{4}} n_{\mu} n_{\nu}
$$

The only null eigenvectors are $\dot{X}$ and $X_{A}$. The quadratic model (46) is representative of the generic model (44) as long as this is the case.

The Hamiltonian that generates the dynamics is given by adding the primary constraints (87), 887) to the canonical Hamiltonian (85),

$$
H=H_{c}+\int_{\Sigma}\left(\lambda C+\lambda^{A} C_{A}\right)
$$

with $\lambda, \lambda^{A}$ arbitrary Lagrange multipliers.

There are also secondary constraints. They do not participate in the determination of the dynamics, but they are important as generators of worldvolume reparametrizations. From the conservation in time of the primary constraint (87), we obtain the secondary constraint that the integrand of the canonical Hamiltonian (85) must vanish,

$$
S=\mathcal{H}_{c}=0 .
$$

where we have defined $H_{c}=\int_{\Sigma} \mathcal{H}_{c}$. Just as in the DNG case, this was to be expected on the basis of reparametrization invariance. Conservation in time of the other primary constraint (88) produces the additional secondary constraint

$$
S_{A}=p \cdot X_{A}+P \cdot \partial_{A} \dot{X}=0 .
$$

There are no tertiary constraints. The constraint algebra of the four constraints is in involution; they are first class. The full Poisson algebra of the constraints is given in Appendix B.

The constraint (92) is the generator of diffeomorphisms tangential to $\Sigma$, as it can be verified directly considering its Poisson bracket with the phase space variables (see Appendix B). The vanishing of the canonical Hamiltonian, the secondary constraint (91), generates diffeomorphisms out of $\Sigma$ onto the worldvolume $m$. Again, this can be verified by considering the Poisson bracket of $S$ with the phase space variables. This can be read out from the Hamilton's equations of motion given below in Sect. 7. Also the effect of the primary constraints on the phase space variables is listed in Appendix B.

The number of physical degrees of freedom for the action (46) is equal to $1 / 2[4(d+2)-4(d+1)]=2$. There are two physical degrees of freedom associated with the single normal to the worldvolume. For higher co-dimension there will be two physical degree of freedom for each normal. This is twice the number of physical degrees of freedom in the DNG model. The appearance of extra degrees of freedom is a generic feature of higher derivative theories. 


\section{Hamilton's equations}

In this section we consider the Hamilton equations for the model (44), generated by the Hamiltonian (90). We check explicitly that, at the end of the day, Hamilton's equations correctly reproduce the equations of motion.

As we pointed out in Sect. 3, the first Hamilton equation $\dot{X}=\delta H / \delta p$ is an identity, since the only dependence on $p$ is thought the $p \cdot \dot{X}$ term in $H_{c}$. The second Hamilton equation reproduces the form of the momenta $P$ given by (69), and furthermore identifies the form of the Lagrange multipliers $\lambda, \lambda^{A}$ in terms of the canonical variables. We obtain

$\ddot{X}=\frac{\delta H}{\delta P}=\frac{N^{3}}{\sqrt{h}} P+2 N^{A} \partial_{A} \dot{X}+\left(N^{2} h^{A B}-N^{A} N^{B}\right) \mathcal{D}_{A} \mathcal{D}_{B} X+\lambda \dot{X}+\lambda^{A} X_{A}$.

To see that this gives (69), the quickest way is to contract with the worldvolume normal $n$, obtaining

$$
\begin{aligned}
P \cdot n & =\frac{\sqrt{h}}{N^{3}}\left[-K_{00}+2 N^{A} K_{0 A}+\left(N^{2} h^{A B}-N^{A} N^{B}\right) K_{A B}\right. \\
& =\frac{\sqrt{h}}{N} K,
\end{aligned}
$$

where we have used (82). Contraction of (93) with $\dot{X}$ and $X_{A}$, and use of the constraints identifies the Lagrange multipliers as (see Appendix A)

$$
\begin{aligned}
& \lambda=\mathcal{D}_{A} N^{A}-\frac{N^{2}}{\sqrt{h}} \eta^{a} \nabla_{a}\left(\frac{\sqrt{h}}{N}\right) \\
& \lambda^{A}=\frac{N^{2}}{\sqrt{h}} \eta^{a} \nabla_{a}\left(\frac{N^{A} \sqrt{h}}{N}\right)-N^{A} \mathcal{D}_{B} N^{B}+N h^{A B} \mathcal{D}_{B} N .
\end{aligned}
$$

Note that these relationships involve derivatives of the lapse function, the shift vector and the $\Sigma$ volume element. They also involve mixing: $\lambda$ and $\lambda^{A}$ depend on both $N$ and $N^{A}$. Gauge fixing will amount to a specification of $\lambda$ and $\lambda_{A}$ or, equivalently, $N$ and $N^{A}$. Clearly, this will be qualitatively very different from the familar DNG case.

Let us turn to the third Hamilton equation. This identifies the form of the momenta $p$ as given by (70). We have

$$
\begin{aligned}
\dot{P}= & -\frac{\delta H}{\delta \dot{X}}=-p+\frac{3}{2} \frac{N^{2}}{\sqrt{h}} P^{2} \eta-2 h^{A B}\left(P \cdot \partial_{A} \dot{X}\right) X_{B}+2 \partial_{A}\left(N^{A} P\right) \\
& +2 N h^{A B}\left(P \cdot \mathcal{D}_{A} \mathcal{D}_{B} X\right) \eta+2 N^{A} h^{B C}\left(P \cdot \mathcal{D}_{A} \mathcal{D}_{B} X\right) X_{C}-\lambda P .
\end{aligned}
$$

To see that this expression coincides with (70), first note that

$$
\begin{aligned}
\frac{3}{2} \frac{N^{2}}{\sqrt{h}} P^{2} \eta & -2 h^{A B}\left(P \cdot \partial_{A} \dot{X}\right) X_{B}+2 N h^{A B}\left(P \cdot \mathcal{D}_{A} \mathcal{D}_{B} X\right) \eta+2 N^{A} h^{B C}\left(P \cdot \mathcal{D}_{A} \mathcal{D}_{B} X\right) X_{C} \\
& =\sqrt{h}\left[\left(\frac{3}{2} K^{2}-2 K h^{A B} K_{A B}\right) \eta+\frac{2}{N}\left(h^{A B} K K_{0 A}-N^{A} h^{B C} K K_{A C}\right) X_{B}\right] \\
& =-\frac{1}{2} \sqrt{h} K^{2} \eta+2 \sqrt{h} K K^{a b} \eta_{a} X_{b},
\end{aligned}
$$

where we have used (32), (33). Therefore we find

$$
p=-\frac{1}{2} \sqrt{h} K^{2} \eta+2 \sqrt{h} K K_{a b} \eta_{a} X_{b}-\dot{P}+2 \partial_{A}\left(N^{A} P\right)-\lambda P,
$$


and inserting the form of the Lagrange mutiplier (96) we recover (70) for $p$.

The fourth Hamilton equation is

$$
\dot{p}=-\frac{\delta H}{\delta X} .
$$

Using the explicit form of the momenta and of the Lagrange multipliers, this equation ought to reproduce the equation of motion (62). To show this turns out to be a little more involved than one may have expected. First of all, in the variation of the Hamiltonian with respect to $X$, it is necessary to recognize that, besides the obvious dependence on $X$ of the metric induced on $\Sigma, h_{A B}$, there is also a dependence implicit in the lapse function $N$ and the shift vector $N^{A}$. Holding $\dot{X}$ fixed, we have that the unit normal to $\Sigma$ onto $m$ varies with respect to $X$ as

$$
\delta \eta=-\left(\eta \cdot \partial_{A} \delta X\right) h^{A B} X_{B}-\frac{N^{A}}{N}\left(n \cdot \partial_{A} \delta X\right) n .
$$

It implies that the variation of the lapse function with respect to $X$, again holding $\dot{X}$ fixed, is

$$
\delta N=N^{A}\left(\eta \cdot \partial_{A} \delta X\right) .
$$

For the variation of the shift vector with respect to the embedding functions $X$ for $\Sigma$, we find

$$
\delta N^{A}=N h^{A B}\left(\eta \cdot \partial_{B} \delta X\right)-N^{B} h^{A C}\left(X_{C} \cdot \partial_{B} \delta X\right) .
$$

Consider now the variation of the Hamiltonian, holding $\dot{X}, p, P$ fixed. We have

$$
\begin{aligned}
\delta H & =\int_{\Sigma}\left\{\left[-\frac{N^{3}}{2 \sqrt{h}} P^{2} h^{A B}-2 N^{A} h^{B C}\left(P \cdot \partial_{C} \dot{X}\right)-2 N^{2} h^{A C} h^{B D}\left(P \cdot \mathcal{D}_{C} \mathcal{D}_{D} X\right)\right.\right. \\
& \left.+2 N^{A} N^{D} h^{B C}\left(P \cdot \mathcal{D}_{C} \mathcal{D}_{D} X\right)\right]\left(X_{B} \cdot \partial_{A} \delta X\right) \\
& +\left[\frac{3 N^{3}}{2 \sqrt{h}} N^{A} P^{2}+2 N h^{A B}\left(P \cdot \partial_{B} \dot{X}\right)+4 N N^{(A} h^{B) C}\left(P \cdot \mathcal{D}_{B} \mathcal{D}_{C} X\right)\right. \\
& \left.-4 N N^{(B} h^{A) C}\left(P \cdot \mathcal{D}_{C} \mathcal{D}_{B} X\right)\right]\left(\eta \cdot \partial_{A} \delta X\right) \\
& \left.+\left(N^{2} h^{A B}-N^{A} N^{B}\right)\left(P \cdot \mathcal{D}_{A} \mathcal{D}_{B} \delta X\right)+\lambda^{A} P \cdot \partial_{A} \delta X\right\} .
\end{aligned}
$$

We integrate by parts and, disregarding a total spatial divergence, we carry out the functional derivative in (100), so that we obtain

$$
\begin{aligned}
\dot{p} & =-\partial_{A}\left\{\left[\frac{N^{3}}{2 \sqrt{h}} P^{2} h^{A B}+2 N^{A} h^{B C}\left(P \cdot \partial_{C} \dot{X}\right)+2 N^{2} h^{A C} h^{B D}\left(P \cdot \mathcal{D}_{C} \mathcal{D}_{D} X\right)\right.\right. \\
& \left.-2 N^{A} N^{D} h^{B C}\left(P \cdot \mathcal{D}_{C} \mathcal{D}_{D} X\right)\right] X_{B} \\
& +\left[-\frac{3 N^{3}}{2 \sqrt{h}} N^{A} P^{2}-2 N h^{A B}\left(P \cdot \partial_{B} \dot{X}\right)-4 N N^{(A} h^{B) C}\left(P \cdot \mathcal{D}_{B} \mathcal{D}_{C} X\right)\right. \\
& \left.+4 N N^{(B} h^{A) C}\left(P \cdot \mathcal{D}_{C} \mathcal{D}_{B} X\right)\right] \eta \\
& \left.+\partial_{B}\left[\left(N^{2} h^{A B}-N^{A} N^{B}\right) P\right]-\lambda^{A} P\right\} .
\end{aligned}
$$

Now, we need to recognize that the part proportional to the shift vector $N^{A}$ is related to the momenta $p$ via (96), so that the equations of motion can be written in canonical 
form as

$$
\begin{aligned}
\dot{p} & =\partial_{A}\left\{N^{A} p+N^{A} \dot{P}-2 N^{A} \partial_{B}\left(N^{B} P\right)+\lambda N^{A} P+\lambda^{A} P+\mathcal{D}_{B}\left[\left(N^{2} h^{A B}-N^{A} N^{B}\right) P\right]\right. \\
& +N^{2}\left[\frac{N}{2 \sqrt{h}} P^{2} h^{A B}+2 h^{A C} h^{B D}\left(P \cdot \mathcal{D}_{C} \mathcal{D}_{D} X\right)\right] X_{B} \\
& \left.+2 N\left[N^{C} h^{A B}\left(P \cdot \mathcal{D}_{B} \mathcal{D}_{C} X\right)-\left(P \cdot \partial_{B} \dot{X}\right) h^{A B}\right] \eta\right\} .
\end{aligned}
$$

It is not at all obvious that this form of the equations of motion coincides with the covariant expression (62). In order to be able to compare the two, we decompose the (densitized) Euler-Lagrange derivative in (62) with respect to the worldvolume basis $\left\{\dot{X}, X_{A}\right\}$. This gives

$$
\begin{aligned}
\sqrt{-g} E & =\partial_{0}\left\{N \sqrt{h}\left[-\frac{1}{2} g^{0 a} K^{2} X_{a}+2 g^{0 c} K K^{a}{ }_{c} X_{a}-g^{0 a} \nabla_{a}(K n)\right]\right\} \\
& +\partial_{A}\left\{N \sqrt{h}\left[-\frac{1}{2} g^{A a} K^{2} X_{a}+2 g^{A c} K K^{a}{ }_{c} X_{a}-g^{A a} \nabla_{a}(K n)\right]\right\},
\end{aligned}
$$

and we use (32), (33) for $g^{0 a}, g^{A b}$, to obtain

$$
\begin{aligned}
\sqrt{-g} E & =\partial_{0}\left\{\sqrt{h}\left[\frac{1}{2} K^{2} \eta-2 \eta^{c} K K_{c}^{a} X_{a}+\eta^{a} \nabla_{a}(K n)\right]\right\} \\
& +\partial_{A}\left\{\sqrt { h } \left[-\frac{N}{2} K^{2} h^{A B} X_{A}-\frac{1}{2} N^{A} K^{2} \eta+2 N h^{A B} K K_{B}^{a}{ }_{B} X_{a}+2 N^{A} \eta^{c} K K_{a}{ }^{c} X_{c}\right.\right. \\
& \left.\left.-N h^{A B} \mathcal{D}_{B}(K n)-N^{A} \eta^{a} \nabla_{a}(K n)\right]\right\} .
\end{aligned}
$$

The argument of the time derivative is related to $p$, using (70), and the same combination appears in the spatial divergence, in the terms proportional to $N^{A}$, so that

$$
\begin{aligned}
\sqrt{-g} E & =-\dot{p}+\partial_{0} \partial_{A}\left(N^{A} P\right)+\partial_{A}\left[N^{A} p-N^{A} \partial_{B}\left(N^{B} P\right)-\frac{N \sqrt{h}}{2} K^{2} h^{A B} X_{B}\right. \\
& -N \sqrt{h} h^{A B} \mathcal{D}_{B}(K n)-2 N \sqrt{h} K K^{A a} \eta_{a} \eta+2 N \sqrt{h} K K^{A B} X_{B} \\
& \left.-N \sqrt{h} \mathcal{D}^{A}(K n)\right],
\end{aligned}
$$

which in terms of the phase space variables takes the form

$$
\begin{aligned}
\sqrt{-g} E= & -\dot{p}+\partial_{A}\left\{\partial_{0}\left(N^{A} P\right)+N^{A} p-N^{A} \partial_{B}\left(N^{B} P\right)-N \sqrt{h} h^{A B} \mathcal{D}_{B}(K n)\right. \\
& -N^{2}\left[\frac{1}{2} \frac{N}{\sqrt{h}} P^{2} h^{A B}+2 h^{A C} h^{B D}\left(P \cdot \mathcal{D}_{C} \mathcal{D}_{D} X\right)\right] X_{B} \\
& +2 N\left[\left(P \cdot \partial_{B} \dot{X}\right) h^{A B}-N^{B}\left(p \cdot \mathcal{D}_{A} \mathcal{D}_{B} X\right)\right] \eta .
\end{aligned}
$$

To see that the vanishing of this expression coincides with the canonical equations of motion (106) it is sufficient to use the explicit form of the Lagrange multipliers (95), (96) in (106).

\section{Higher co-dimension}

In order to minimize the already vexing amount of formalism, so far we have restricted our attention to the case where the worldvolume $m$ is a timelike hypersurface in spacetime, with a single normal vector field. In this section, we lift this restriction, and 
consider arbitrary co-dimension. As it becomes clear soon enough, the modification in the Hamiltonian formulation consists basically of a decoration with normal indices every time a normal vector field appears.

In a fixed Minkowski background of dimension $d+N+1$, for a relativistic extended object of dimension $d$, we have $N$ unit spacelike normal vector fields $n^{i}$ $(i, j, \ldots=1,2, \cdots, N)$, defined implicitly by $n^{i} \cdot X_{a}=0$ and $n^{i} \cdot n^{j}=\delta^{i j}$, with $\delta^{i j}$ the Kronecker delta. The Gauss-Weingarten equations take the form

$$
\begin{aligned}
& \nabla_{a} X_{b}=-K_{a b}{ }^{i} n_{i}, \\
& \nabla_{a} n^{i}=K_{a c} g^{b c} X_{c}+\omega_{a}^{i j} n_{j} .
\end{aligned}
$$

There are $N$ extrinsic curvature tensors, one along each normal, and $N$ mean extrinsic curvatures, $K^{i}=g^{a b} K_{a b}{ }^{i}$. The new structure is the normal connection $\omega_{a}^{i j}=-\omega_{a}^{j i}$ associated with the $O(N)$ invariance under rotations of the normal vector fields. We use it to define the $O(N)$ covariant derivative $\tilde{\nabla}_{a}=\delta^{i j} \nabla_{a}-\omega_{a}^{i j}$. As long as one is concerned with quantities invariant under rotations of the normals, the normal connection will appear only in intermediate steps of the calculations.

We consider an action that depends on the $N$ extrinsic curvatures, generalizing (44) to

$$
S[X]=\int_{m} \sqrt{-g} L\left(g^{a b}, K_{a b}{ }^{i}\right),
$$

which, besides the requirements of Poincaré and reparametrization invariance, is also subject to the requirement of invariance under rotations of the normal fields, such as e.g. the higher co-dimension version of the quadratic model (46),

$$
S[X]=\frac{1}{2} \int_{m} \sqrt{-g} K^{i} K_{i} .
$$

For the case of a relativistic string, this action was proposed by Polyakov, and independently by Kleinert [4] [5].

Let us describe briefly how the Hamiltonian formulation generalizes to higher co-dimension. For an action of the form (113), the momenta are

$$
\begin{gathered}
P=-\frac{\sqrt{h}}{N} \eta_{b} L^{a b}{ }_{i} \eta_{a} n^{i}, \\
p=-\sqrt{h}\left[L \eta-2 \eta_{b} H^{a b} X_{a}+\eta_{b} \nabla_{a}\left(L_{i}^{a b} n^{i}\right)\right]+\partial_{A}\left[N^{A} P-\sqrt{h} \eta^{a} L_{a b i} X^{b A} n^{i}\right] .
\end{gathered}
$$

where $L_{i}^{a b}=\partial L / \partial K_{a b}{ }^{i}$. For the quadratic model (114), the momenta become

$$
\begin{aligned}
& P=\frac{\sqrt{h}}{N} K_{i} n^{i} \\
& p=-\sqrt{h}\left[\frac{1}{2} K^{i} K_{i} \eta-2 \eta_{b} K^{i} K^{a b}{ }_{i} X_{a}+\eta^{a} \nabla_{a}\left(K_{i} n^{i}\right)\right]+\partial_{A}\left(N^{A} P\right)
\end{aligned}
$$

The canonical Hamiltonian is unchanged with respect to the co-dimension one case analyzed in Sect. 6, and it is given by (85), also the phase space constraints, their algebra and the Hamilton's equations are unchanged. What will change is the counting of the physical degrees of freedom of the theory; in the non-degenerate case there will be two physical dgrees of freedom for each normal. 


\section{Concluding remarks}

In this paper, we have presented a Hamiltonian formulation of the dynamics of a relativistic extended object propagating in Minkowski spacetime described by a local action that depends on the extrinsic curvature of its worldvolume. We have focused our attention on a model quadratic in the mean extrinsic curvature, in the special case of an object of dimension $d$ propagating in a Minkowski background of dimension $d+2$. However, this is not a real restriction: an arbitrary dependence on curvature and a higher co-dimension requires only minimal and straightforward modifications. (The generalization to an arbitrary background can be carried out along similar lines.) This is true as long as the structure of the primary constraints is the same as in the quadratic case. One obvious case where this does not happen is for models with a linear dependence on the acceleration, such as the Einstein-Hilbert action. Such special cases will be considered elsewhere [37]. A less obvious special case is provided by the 'gonihedric' string model, with action [39]

$$
S[X]=\int_{m} \sqrt{-g} \sqrt{K^{i} K_{i}} .
$$

For this model, there are additional primary constraints and the study of its Hamiltonian formulation is in progress [40].

\section{Acknowledgments}

JG thanks Denjoe O' Connor for hospitality during his stay at DIAS. He also acknowledges partial support from DGAPA-PAPIIT grant IN114302. ER acknowledges partial support from CONACyT under grant CO1-41639 and PROMEP2003.

\section{APPENDIX A: USEFUL FORMULAE}

In this Appendix we collect some useful formulae about the geometries of the worldvolume $m$, its spatial slice $\Sigma$, and their relationships. The Gauss-Weingarten equations for the worldvolume $m$ are

$$
\begin{aligned}
& \nabla_{a} X_{b}=-K_{a b} n, \\
& \nabla_{a} n=K_{a b} g^{b c} .
\end{aligned}
$$

Their integrability conditions give the Gauss-Codazzi-Mainardi equations for the worldvolume, in a Minkowski background,

$$
\begin{aligned}
\mathcal{R}_{a b c d} & =K_{a c} K_{b d}-K_{a d} K_{b c}, \\
\nabla_{a} K_{b c} & =\nabla_{b} K_{a c} .
\end{aligned}
$$

Contractions with the inverse induced metric $g^{a b}$ gives

$$
\begin{aligned}
& \mathcal{R}_{a b}=K K_{a b}-K_{a}{ }^{c} K_{b c}, \\
& \mathcal{R}=K^{2}-K^{a b} K_{a b}, \\
& \nabla_{b} K^{b}{ }_{a}=\nabla_{a} K .
\end{aligned}
$$


The Gauss-Weingarten equations for $\Sigma$ seen as embedded in the worldvolume $m$ are

$$
\begin{aligned}
& \mathcal{D}_{A} X_{B}=k_{A B} \eta, \\
& \mathcal{D}_{A} \eta=k_{A B} h^{B C} X_{C},
\end{aligned}
$$

where the spatial tensor $k_{A B}$ is the extrinsic curvature of $\Sigma$ as embedded in the worldvolume $m$,

$$
k_{A B}=-\eta \cdot \mathcal{D}_{A} \mathcal{D}_{B} X .
$$

The projection of the Gauss-Weingarten equations along the basis $\left\{\eta, n, X_{A}\right\}$ adapted to $\Sigma$ gives

$$
\begin{array}{ll}
\ddot{X} & =-K_{00} n+\left(\dot{N}_{A}+N \mathcal{D}_{A} N-N^{B} \mathcal{D}_{A} N_{B}\right) h^{A C} X_{C} \\
& +\left(\dot{N}+N^{A} \mathcal{D}_{A} N+N^{A} N^{B} k_{A B}\right) \eta \\
\partial_{A} \dot{X} & =\left(\mathcal{D}_{A} N+N^{B} k_{A B}\right) \eta+\left(\mathcal{D}_{A} N_{B}+N k_{A B}\right) h^{B C} X_{C}-K_{0 A} n, \\
\mathcal{D}_{A} \mathcal{D}_{B} X & =k_{A B} \eta-K_{A B} n \\
\dot{\eta} & =\left(\mathcal{D}_{A} N+N^{B} k_{A B}\right) h^{A C} X_{C}-\frac{1}{N}\left(K_{00}-N^{A} K_{0 A}\right) n, \\
\mathcal{D}_{A} \eta \quad & =k_{A B} h^{B C} X_{C}-\frac{1}{N}\left(K_{0 A}-N^{B} K_{A B}\right) n, \\
\dot{n} & =K_{0 A} h^{A B} X_{B}-\frac{1}{N}\left(K_{00}-N^{A} K_{0 A}\right) \eta, \\
\mathcal{D}_{A} n & =K_{A B} h^{B C} X_{C}-\frac{1}{N}\left(K_{0 A}-N^{B} K_{A B}\right) \eta .
\end{array}
$$

Where $K_{00}, K_{0 A}, K_{A B}$ are the projections of the extrinsic curvature tensor $K_{a b}$, defined in (79), (80), (81).

We record also the time derivative of the spatial metric $h_{A B}$ and its determinant,

$$
\begin{aligned}
& \dot{h}_{A B}=2 \mathcal{D}_{(A} N_{B)}+2 N k_{A B}, \\
& \dot{h}=2 h\left(N h^{A B} k_{A B}+\mathcal{D}_{A} N^{A}\right) .
\end{aligned}
$$

\section{APPENDIX B: POISSON CONSTRAINTS ALGEBRA}

In this Appendix we collect the Poisson constraints algebra first for the DNG model, and then for the model quadratic in the mean extrinsic curvature (46).

Consider first the constraints (23), (22) for the DNG model. We define the phase space functions

$$
\begin{aligned}
C_{\lambda} & =\int_{\Sigma} \lambda\left(p^{2}+\mu^{2} h\right), \\
V_{\vec{\lambda}} & =\int_{\Sigma} \lambda^{A} p \cdot X_{A} .
\end{aligned}
$$

They satisfy the Poisson algebra

$$
\begin{aligned}
& \left\{C_{\lambda}, C_{\lambda^{\prime}}\right\}=V_{\overrightarrow{\lambda *}}, \\
& \left\{V_{\vec{\lambda}}, C_{\lambda}\right\}=C_{\mathcal{L}_{\vec{\lambda}} \lambda}, \\
& \left\{V_{\vec{\lambda}}, V_{\overrightarrow{\lambda^{\prime}}}\right\}=V_{\left[\vec{\lambda}, \overrightarrow{\lambda^{\prime}}\right]},
\end{aligned}
$$


where in (139) we have defined $\lambda^{* A}=4 \mu^{2} h h^{A B}\left(\lambda \partial_{B} \lambda^{\prime}-\lambda^{\prime} \partial_{B} \lambda\right)$, and $\mathcal{L}_{\vec{\lambda}}$ denotes the spatial Lie derivative along the vector $\lambda^{A}$. The constraints (23) and (22) are first class.

Next consider the primary constraints (87), 888), and the secondary constraints (91), (92) for the quadratic model (46). We define the phase space functions

$$
\begin{aligned}
C_{\lambda} & =\int_{\Sigma} \lambda P \cdot \dot{X}, \\
V_{\vec{\lambda}} & =\int_{\Sigma} \lambda^{A} P \cdot X_{A}, \\
S_{\Lambda} & =\int_{\Sigma} \Lambda \mathcal{H}_{c} \\
& =\int_{\Sigma} \Lambda\left[p \cdot \dot{X}+\frac{N^{3}}{2 \sqrt{h}} P^{2}+2 N^{A} P \cdot \partial_{A} \dot{X}+\left(N^{2} h^{A B}-N^{A} N^{B}\right) P \cdot \mathcal{D}_{A} \mathcal{D}_{B} X\right] . \\
T_{\vec{\Lambda}} & =\int_{\Sigma} \Lambda^{A}\left(p \cdot X_{A}+P \cdot \partial_{A} \dot{X}\right) .
\end{aligned}
$$

The Poisson constraint algebra of the primary constraints is

$$
\begin{aligned}
& \left\{C_{\lambda}, C_{\lambda^{\prime}}\right\}=0, \\
& \left\{C_{\lambda}, V_{\vec{\lambda}}\right\}=V_{\overrightarrow{\lambda^{\prime}}}, \\
& \left\{V_{\vec{\lambda}}, V_{\overrightarrow{\lambda^{\prime}}}\right\}=0,
\end{aligned}
$$

where $\lambda^{\prime} A=\lambda \lambda^{A}$ in (143).

The Poisson constraint algebra between the primary and secondary constraints, which serves to identify the latter, is

$$
\begin{aligned}
& \left\{C_{\lambda}, S_{\Lambda}\right\}=-S_{\lambda \Lambda}-S_{\mathcal{L}_{\overrightarrow{N^{\prime}}} \lambda}, \\
& \left\{C_{\lambda}, T_{\vec{\Lambda}}\right\}=-C_{\mathcal{L}_{\vec{\Lambda}} \lambda}, \\
& \left\{V_{\vec{\lambda}}, S_{\Lambda}\right\}=-T_{\overrightarrow{\Lambda^{\prime}}}+C_{\mathcal{L}_{\vec{\lambda}} \Lambda}-V_{\overrightarrow{\lambda^{\prime}}}, \\
& \left\{V_{\vec{\lambda}}, T_{\vec{\Lambda}}\right\}=V_{[\vec{\lambda}, \vec{\Lambda}]},
\end{aligned}
$$

where in 145) we have defined $N^{\prime A}=2 \Lambda N^{A}$, in 147) $\Lambda^{\prime A}=\Lambda \lambda^{A}$, and $\lambda^{\prime A}=$ $2 \Lambda N^{B} \mathcal{D}_{B} \lambda^{A}$.

Finally, the Poisson algebra of the secondary constraints is

$$
\begin{aligned}
& \left\{S_{\Lambda}, S_{\Lambda^{\prime}}\right\}=C_{\widetilde{\lambda}}, \\
& \left\{S_{\Lambda}, T_{\vec{\Lambda}}\right\}=-S_{\mathcal{L}_{\vec{\Lambda}} \Lambda}+V_{\overrightarrow{\hat{\lambda}}}, \\
& \left\{T_{\vec{\Lambda}}, T_{\overrightarrow{\Lambda^{\prime}}}\right\}=T_{\left[\vec{\Lambda}, \overrightarrow{\Lambda^{\prime}}\right]} .
\end{aligned}
$$

where in (149) we have defined $\tilde{\lambda}=\left(N^{2} h^{A B}-N^{A} N^{B}\right)\left(\Lambda \mathcal{D}_{A} \mathcal{D}_{B} \Lambda^{\prime}-\Lambda^{\prime} \mathcal{D}_{A} \mathcal{D}_{B} \lambda\right)$, and in (150) $\hat{\lambda}^{A}=\Lambda\left(N^{2} h^{B C}-N^{B} N^{C}\right)\left(\mathcal{D}_{B} \mathcal{D}_{C} \Lambda^{A}+\Lambda^{D} h^{E A} R_{B E C D}\right) . \quad R_{B E C D}$ is the Riemann tensor on $\Sigma$. The constraints are first class. For the special case of a relativistic string there is a considerable simplification in the form of the structure functions of the algebra.

We record also the action of the constraints on the phase space variables

$$
\begin{aligned}
& \left\{C_{\lambda}, X\right\}=0, \\
& \left\{C_{\lambda}, \dot{X}\right\}=-\lambda \dot{X}, \\
& \left\{C_{\lambda}, p\right\}=0, \\
& \left\{C_{\lambda}, P\right\}=\lambda P
\end{aligned}
$$




$$
\begin{aligned}
& \left\{V_{\vec{\lambda}}, X\right\}=0, \\
& \left\{V_{\vec{\lambda}}, \dot{X}\right\}=-\mathcal{L}_{\vec{\lambda}} X, \\
& \left\{V_{\vec{\lambda}}, p\right\}=-\mathcal{L}_{\vec{\lambda}} P, \\
& \left\{V_{\vec{\lambda}}, P\right\}=0, \\
& \left\{T_{\vec{\Lambda}}, X\right\}=-\mathcal{L}_{\vec{\Lambda}} X, \\
& \left\{T_{\vec{\Lambda}}, \dot{X}\right\}=-\mathcal{L}_{\vec{\Lambda}} \dot{X}, \\
& \left\{T_{\vec{\Lambda}}, p\right\}=-\mathcal{L}_{\vec{\Lambda}} p, \\
& \left\{T_{\vec{\Lambda}}, P\right\}=-\mathcal{L}_{\vec{\Lambda}} P .
\end{aligned}
$$

We refrain from writing down the Poisson bracket of the secondary constraint $S_{\Lambda}$ on the phase space variables because it can be obtained easily from the calculations that lead to the Hamilton's equations in Sect. 7.

\section{References}

[1] Dirac P A M 1962 Proc. R. Soc. London Ser. A 26857

[2] Nambu Y 1970 Copenhagen Summer Symposium (unpublished)

[3] Goto T 1971 Prog. Theor. Phys. 461560

[4] Polyakov A M 1986 Nucl. Phys. B 268406

[5] Kleinert H 1986 Phys. Lett. B 174335

[6] Maeda K and Turok N 1988 Phys. Lett. B 202376

[7] Gregory R 1988 Phys. Lett. B 206199

[8] Garfinkle D and Gregory R 1990 Phys. Rev. D 411889

[9] Carter B and Gregory R 1995 Phys. Rev. D 515839

[10] Randall L and Sundrum R 1999 Phys. Rev. Lett. 83 3370; 834690

[11] Rogers C and Schief W K 2002 Backlund and Darboux Transformations (Cambridge: Cambridge University Press)

[12] Ambjorn J, Durhuus B and Jonsson T 1997 Quantum geometry - A statistical field theory approach (Cambridge; Cambridge University Press )

[13] Bowick M and Travesset A 2001 Phys. Repts. 344255

[14] Nelson D, Piran T and Weinberg S eds 1989 Statistical Mechanics of Membranes and Surfaces vol. 5 (Proceedings of the Jerusalem Winter School for Theoretical Physics) (Singapore: World Scientific);

[15] Seifert U 1997 Adv. in Phys. 4613

[16] Nesterenko V V and Nguyen Suan Han 1988 Int. J. Mod. Phys. A 32315

[17] Nesterenko V V 1989 J. Phys. A: Math. and Gen. 221673

[18] Polyakov A M 1987 Gauge Fields and Strings (Reading UK: Harwood Academic)

[19] Hoppe J 1994 hep-th/9407103

[20] Hoppe J 1995 hep-th/9503069

[21] Hoppe J and Ratiu T 1997 Class. Quant. Grav. 14 L45

[22] Bordemann M and Hoppe J 1998 J. Math. Phys. 39683

[23] Cartas-Fuentevilla R 2002 Phys. Lett. B $536283 ; 536289$

[24] Carter B 2003 Int. J. Theor. Phys. 421317

[25] Capovilla R and Guven J 1995 Phys. Rev. D 516736

[26] Carter B 2001 Int. J. Theor. Phys. 402099

[27] Wald R 1984 General Relativity (Chicago: Chicago Univ. Press)

[28] Ostrogradski M 1850 Mem. de l'Acad. St. Petersbourg 6385

[29] Arreaga G, Capovilla R and Guven J 2000 Annals Phys. 279126

[30] Tolman R C 1949 J. Chem. Phys. 17333

[31] Dolan B P 1990 Z. Phys. C 46317

[32] Smolin L 1998 Phys. Rev. D 576216

[33] Capovilla R, Guven J and Rojas E 2000 Nucl. Phys. B Proc. Suppl. 88337

[34] Regge T and Teitelboim C 1977, in Proceedings of the First Marcel Grossmann Meeting (Amsterdam: North Holland)

[35] Deser S, Pirani F A E and Robinson D C 1976 Phys. Rev. D 143301

[36] Karasik D and Davidson A 2003 Phys. Rev. D 67064012 
[37] Capovilla R, Guven J and Rojas E, in preparation

[38] Capovilla R, Guven J and Rojas E 2002 Class. Quantum Grav. 192277

[39] Manvelian R and Savvidy G 2002 Phys. Lett. B 533138

[40] Capovilla R, Guven J and Rojas E, in preparation 